\title{
FAST SWEEPING DEVICE FOR LASER BUNCH ${ }^{1}$
}

\author{
A. Mikhailichenko, Cornell University, LEPP, Ithaca NY 14853
}

\begin{abstract}
An electro-optical laser sweeping device directs the head and the tail of a laser bunch into different frontal directions, so at some distance, the laser bunch becomes tilted with respect to forward direction. For sweeping of a laser bunch having $300 \mathrm{ps}$ duration up to $10 \mathrm{mrad}$, the voltage drop along the laser bunch must be $\sim 10 \mathrm{kV}$. The repetition rate desirable for this type of device used in laser acceleration or generation secondary backscattered electrons is up to $1 \mathrm{MHz}$. Details of this scheme are described here.
\end{abstract}

\section{INTRODUCTION}

Some time ago we proposed a method of the reduction of surface density of radiation while exciting tiny accelerating structures by laser radiation [1]. The idea is to excite these accelerating structures by focused laser radiation locally through the side openings in these structures. The radiation is focused onto that part of structure, where the accelerated bunch is currently located, so this focused spot follows the bunch while it is moving inside the structure. It was pointed out that the amount of power density reduction defined by the ratio of laser spot size to the length of the accelerating structure. One can see that in this method the time of illumination of each point on the surface is reduced in the same proportion also. We proposed in [1] a method on how to arrange this local excitation by device, which makes sweeping focused laser radiation along the accelerating structure and called this procedure Travelling Laser Focus (TLF). For angular sweep the reduction associated with the number of resolved spots $N_{R}$; so reduction is $\cong 1 / N_{R}$. The number $N_{R}$ is just a ratio of sweep angle to the diffraction angle associated with the sweeping device itself. Basically this number shows how many separated (resolved) focused spots this sweeping device can allocate along the accelerating structure. For laser radiation with the wavelengths $10 \mu m \geq \lambda_{a c} \geq 1 \mu \mathrm{m}$ the number of resolved spots can be within 20-200. This shows that the laser power density distributed over accelerating surface can be up to two hundred times lower than when excitation is going for all structure at the time. One can see also, that lowering of illumination time two hundred times drastically reduces the probability of damaging the surface.

So we believe that TLF is the only way to reach electron-positron ${ }^{2}$ collisions with multi-TeV energy.

In our previous publications [2], [3] we considered some aspects for TLF in general, including a sweeping device. Here we considered a sweeping device in more detail, including analyses for it's practical realization.

We concluded that a sweeping device based on an electro-optical effect (dependence of refractive index on electrical field applied) is mostly adequate to the problem. This device can work up to repetition rate up to few $\mathrm{MHz}$ (or even $\mathrm{GHz}$ ) thereby decreasing requirements to the

\footnotetext{
${ }^{1}$ Electronic version is available at http://www.Ins.cornell.edu/public/CBN/2005/CBN05-6/cbn05-6.pdf.

This work is supported by National Science Foundation.

${ }^{2}$ Also pion-pion, muon-muon, ion-ion and p-p collisions.
} 
bunch population. The last part is very important, taking into account that the luminosity required is a growing function of energy.

\section{TRAVELING LASER FOCUS METHOD}

According to this method, the laser power applied only to the places of the structure, where the accelerated bunch is located at the moment. As the bunch is moving with the speed of light, the laser radiation structure must appear as it is shown in Fig.1 below.

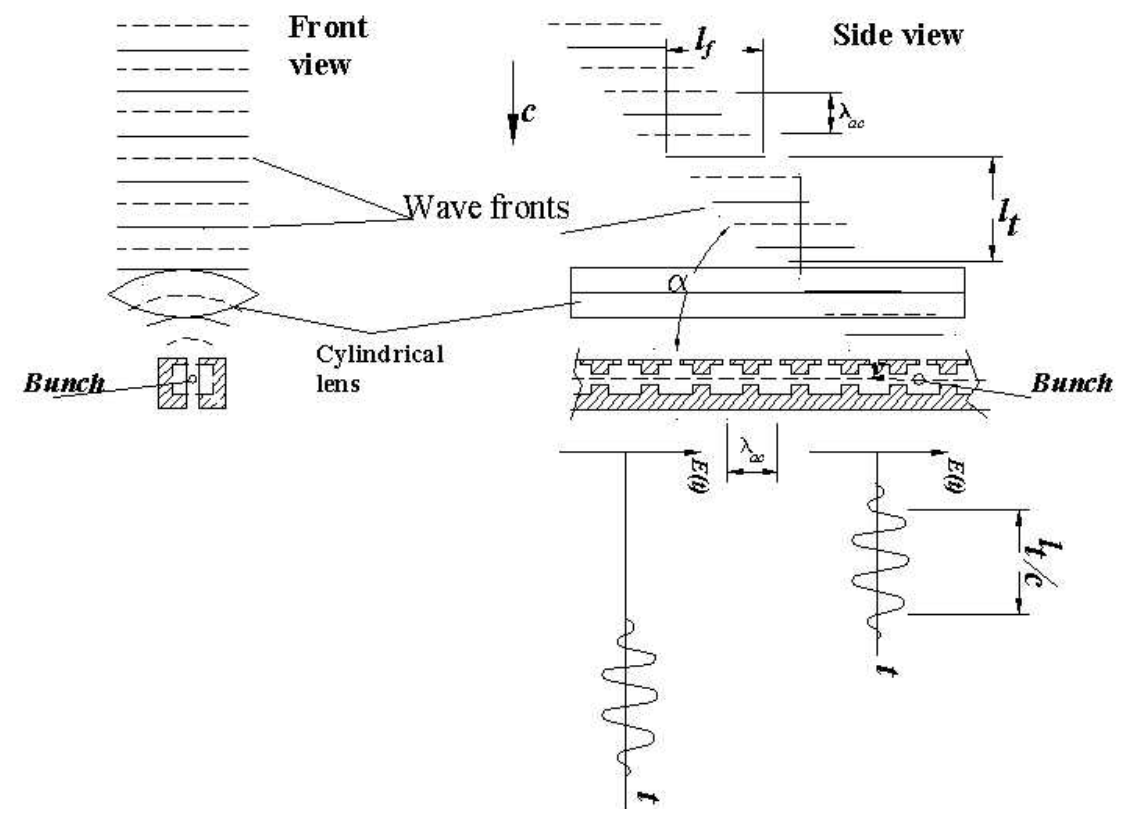

FIGURE 1: The concept of illumination of accelerating structure. The time dependence of the field at two different locations along the structure is represented on the graphs at the bottom. Bunch is running inside structure to the left with a speed $\vec{v}$.

Evidently that the slope angle satisfies the condition $\tan \alpha=c / v$ where $c$ is the speed of light, and $v$ is the speed of the bunch. For relativistic electrons or positrons, the slope angle becomes $\alpha \cong \pi / 4$. Laser radiation applied to every point of structure during $\tau \sim l_{t} / c$, where $l_{t}$ is the instant height of the sloped laser bunch, Fig. 1. The number of accelerating cells excited simultaneously is $\sim l_{f} / \lambda_{a c}$, where $l_{f}$ is a spot size, Fig. 1 . The focal point is following the beam in average. Some mismatches are allowable, as the phase of the laser radiation is synchronized once with the particle's bunch motion. Accelerating cells in a structure separated in longitudinal direction with distance $\lambda_{a c}$, so an electromagnetic field is in phase inside each cell. A cylindrical lens serves for the focusing of laser radiation in a transverse direction.

The method on how to obtain such a laser slope with the help of a sweeping device is represented in Fig.2. A sweeping device uses controllable angular deflection of the laser radiation in time. The device could be characterized by a deflection angle $\vartheta$ and the angle of natural diffraction $\vartheta_{d} \cong \lambda / a$, where $a$-is the aperture of the sweeping device, see Fig.2. The ratio of deflection angle to the diffraction angle is a fundamental measure of the quality for deflecting device. This ratio defines the number of resolved spots (pixels) on the surface of the 
structure, $\quad N_{R}=\vartheta / \vartheta_{d}$. Number of resolved spots $N_{R}$ is an invariant under such transformations, although the deflection angle could be increased by appropriate optics. As we mentioned above, $N_{R}$ value gives the number for the lowering the laser power required for the gradient desired and, also, the number for duty time reduction. The last is important for the structure heating reduction. So it is desirable to have this number as large as possible.

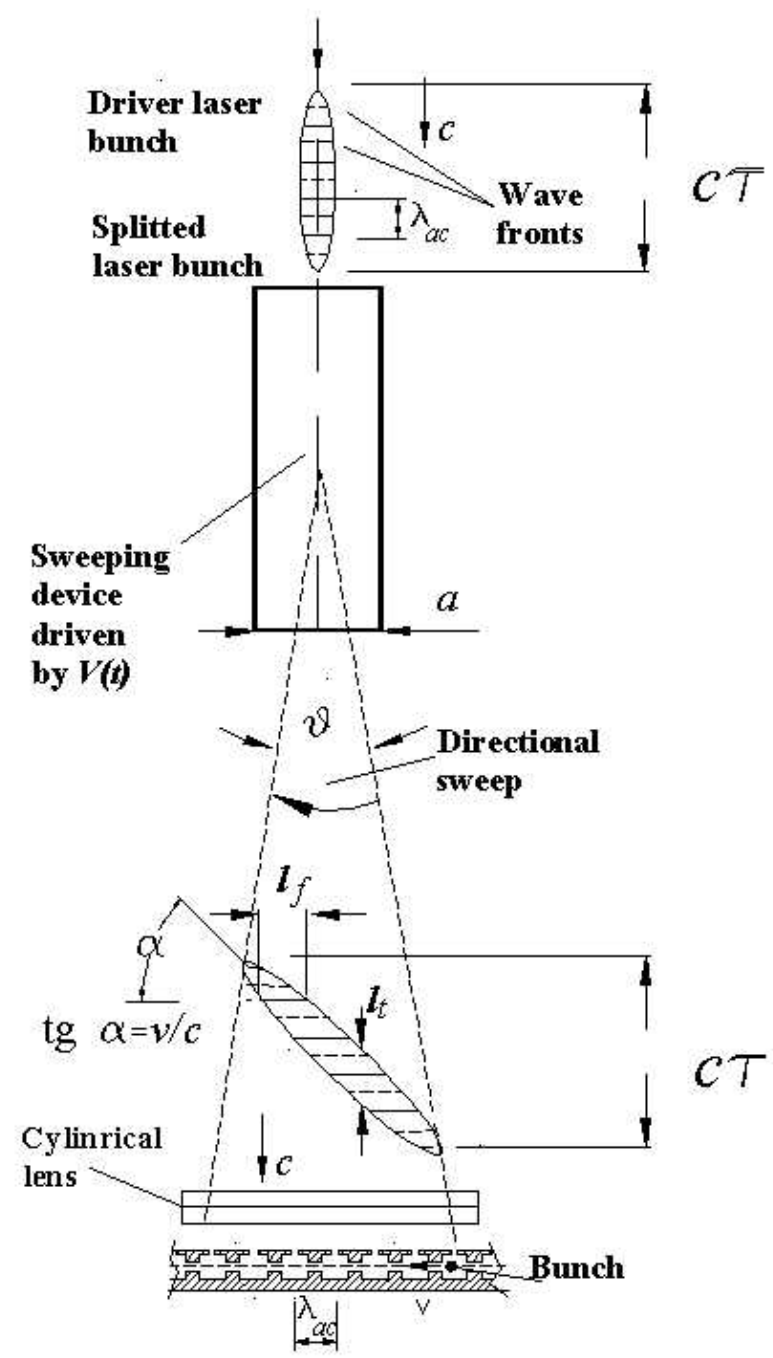

FIGURE 2: Arrangement of traveling laser focus with sweeping device. This device is driven by the time dependent voltage $V(t)$.

Due to this arrangement, all laser pulsed power acts for generation of an accelerating field at the instant particle's location only. Power reduction and shortening of illuminating time is equal numerically to the number of resolved spots (pixels) $N_{R}$, associated with the sweeping device.

The electric field in each point of the laser bunch is perpendicular to the line, connecting this point with the center of the sweeping device. The height of the laser bunch in Fig. 2, which is $c \tau$ does not change, while transverse size is increasing with distance, calculated from the sweeping device. 


\section{ELECTRO OPTICAL EFFECT}

Electro-optical devices use controllable dependence of the refractive index on electrical field strength and direction applied to some crystals.

Indicatrix allows the determination of the refraction index $n$ components for a monochromatic plane wave as a function of their polarization. For the Cartesian coordinate system with the principal axes of a crystal, the Indicatrix can be represented as

$$
\left(\frac{1}{n^{2}}\right)_{1} x^{2}+\left(\frac{1}{n^{2}}\right)_{2} y^{2}+\left(\frac{1}{n^{2}}\right)_{3} s^{2}+2 \cdot\left(\frac{1}{n^{2}}\right)_{4} y s+2 \cdot\left(\frac{1}{n^{2}}\right)_{5} x s+2 \cdot\left(\frac{1}{n^{2}}\right)_{6} x y=1 .
$$

Index runs from 1 to 6 having the following meaning: 1 stands for $x, 2-$ for $y, 3-s, 4-$ for $y s, 5-$ for $x s, 6$ - for $x y$; $s$-longitudinal coordinate.

When an electric field becomes applied to such a crystal, refractive index $n$ changes its value. With abbreviation $\Delta(1 / n)_{i}=1 / n_{i}-1 / n_{0 i}$ this change could be represented as the following

$$
\left(\begin{array}{l}
\Delta\left(1 / n^{2}\right)_{1} \\
\Delta\left(1 / n^{2}\right)_{2} \\
\Delta\left(1 / n^{2}\right)_{3} \\
\Delta\left(1 / n^{2}\right)_{4} \\
\Delta\left(1 / n^{2}\right)_{5} \\
\Delta\left(1 / n^{2}\right)_{6}
\end{array}\right)=\left(\begin{array}{lll}
r_{11} & r_{12} & r_{13} \\
r_{21} & r_{22} & r_{23} \\
r_{31} & r_{32} & r_{33} \\
r_{41} & r_{42} & r_{43} \\
r_{51} & r_{52} & r_{53} \\
r_{61} & r_{62} & r_{63}
\end{array}\right) \times\left(\begin{array}{l}
E_{x} \\
E_{y} \\
E_{s}
\end{array}\right)
$$

where vector $\vec{E}=\left\{E_{x}, E_{y}, E_{s}\right\}$ describes the electrical driving field applied, $r_{i j}-$ are $6 \times 3$ tensor. Accelerating field has a component parallel to $s, E_{s}^{a}$. In components this can be rewritten as the following

$$
1 / n_{i}^{2}=1 / n_{0 i}^{2}+\sum_{j} r_{i j} \cdot E^{j}
$$

So by using (3), the change of reflecting index can be expressed as $\Delta n_{i} \cong\left(\partial n_{i} / \partial E_{j}\right) E^{j}(t)$, where $\partial n_{i} / \partial E_{j}=-n_{0 i}^{3} r_{i j} / 2$ and the net change of refractive index becomes

$$
\Delta n_{i} \cong\left(\partial n_{i} / \partial E_{j}\right) E^{j}(t) \cong-n_{0 j}^{3} r_{i j} E^{j} / 2
$$

Typical values of the $r_{i j}$ are of the order $\cong 10^{-12} \mathrm{~m} / \mathrm{V}$. For example, for GaAs (cubic structure) and for $K D P$ (tetragonal structure) tensor $r_{i j}$ looks respectively

$$
(r)_{i j}=\left(\begin{array}{ccc}
0 & 0 & 0 \\
0 & 0 & 0 \\
0 & 0 & 0 \\
1.5 & 0 & 0 \\
0 & 1.5 & 0 \\
0 & 0 & 1.5
\end{array}\right) \times 10^{-12}[\mathrm{~m} / \mathrm{V}], \quad(r)_{i j}=\left(\begin{array}{ccc}
0 & 0 & 0 \\
0 & 0 & 0 \\
0 & 0 & 0 \\
8.8 & 0 & 0 \\
0 & 8.8 & 0 \\
0 & 0 & 10.5
\end{array}\right) \times 10^{-12}[\mathrm{~m} / V]
$$

For example, substitute the numbers for $\mathrm{ZnTe}$ (transparent for $\mathrm{CO}_{2}$ laser radiation) which has $n_{o} \approx 2.9, \quad r_{41} \cong 4.4 \cdot 10^{-12} \mathrm{~m} / \mathrm{V}, E \cong 10 \mathrm{kV} / \mathrm{cm} \equiv 10^{6} \mathrm{~V} / \mathrm{m}$ one can obtain $\Delta n \cong 10^{-4}$. For KTN (Potassium Tantalite Niobat) crystal $\Delta n \cong 7 \cdot 10^{-3}$ for $\lambda \cong 0.63 \mu \mathrm{m}$ is possible [3].

Let us now see on how this effect can be used for fast sweep of laser bunch. 


\section{ELECTRO OPTICAL DEFLECTION OF LIGHT}

For a prism-based device, Fig.3, change in refraction index yields the change in deflection angle. To arrange such a change, the basements of the prism must be covered by metallic foils and a high voltage applied to them.
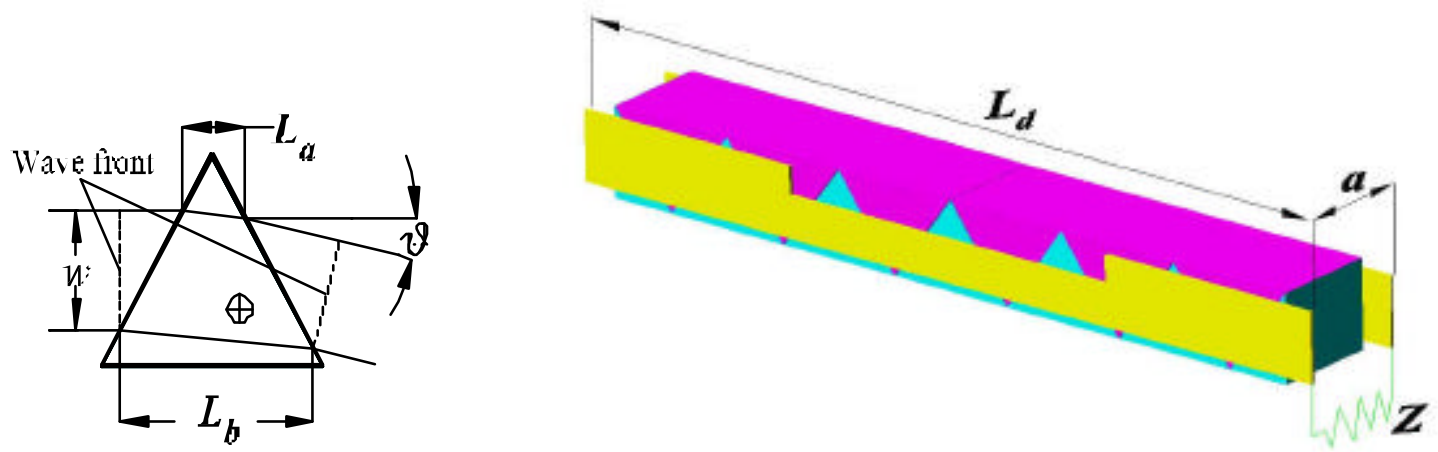

FIGURE 3: The prism deflection device concept, left. Cross marks direction of optical axis. At the rightprisms with oppositely directed optical axes installed in series between two parallel strip-line electrodes, Electromagnetic pulse $E_{x}(t-y / c)$ propagates with laser bunch to the right.

For a prism-based device, Fig.3, left, the deflecting angle is defined by the phase delay across the laser beam front arising from differences in the path lengths in material of the prism having a refractive index $n$

$$
\vartheta \cong n \frac{\left(L_{a}-L_{b}\right)}{w}
$$

where $w \sim a$-is the width of incident laser beam, $L_{a}$ and $L_{b}$-are the distances through which the edges of the laser beam traverse the prism, Fig.3. A change in refractive index value yields a deflection angle change. If the basements of the prism are covered by metallization, then voltage $V(t)$ applied to the metallization will change refractive index as $\Delta n=\Delta n(V(t))$, so

$$
\Delta \vartheta(t) \cong \Delta n(t) \frac{\left(L_{a}-L_{b}\right)}{w} .
$$

The number of resolvable sports for this device $N_{R}$ can be found now as

$$
N_{R} \cong \frac{|\Delta \vartheta|}{\lambda / w}=\frac{\Delta n \cdot\left|L_{a}-L_{b}\right|}{\lambda} \cong \Delta n \frac{l}{\lambda},
$$

where $l=\left|L_{a}-L_{b}\right|$ stands for the prism base length and $a / w \cong 1$ in our case. In any case, a shorter wavelength is preferable from this point of view.

One can see from (6) and (7) that with the increase in optic path difference $l$, both the deflection angle and the number of resolved spots increase also. To increase the last numbers the multiple-prism deflectors were developed, see Fig.3, right. Here neighboring prismatic crystals have oppositely oriented optical axes. These crystals positioned between the strip-line electrodes. In this case the full length of a sweeping device $L_{d}$ serves as $l$ in formulas (7), (8). To be able to sweep short laser bunches, the voltage pulse $V(t)$ is propagating along this strip-line as a traveling wave together with the laser bunch to be swept, as it was proposed in [3]. This gives 
the necessary voltage profile along the laser pulse at $\mathrm{cm}$ distances, what corresponds to the pulse duration $c \tau$, which is $\sim 100$ ps typically [2], [3].

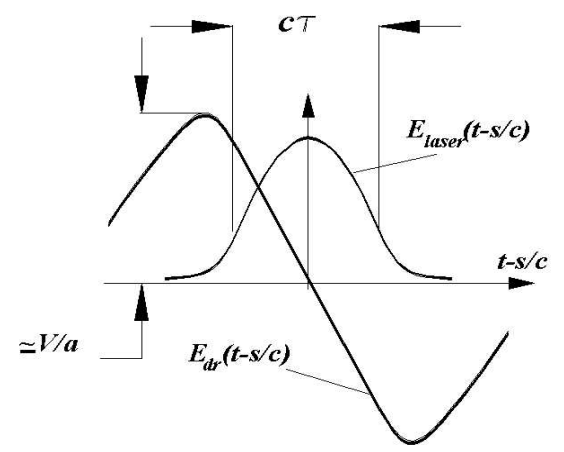

FIGURE 4: Laser pulse and EM driving wave propagating in the same direction.

The deflection angle and the number of resolved spots become now

$$
\Delta \vartheta \cong \Delta n(t) \frac{L_{d}}{w} \cong \frac{L_{d}}{a^{2}} n_{0}^{3} \cdot r_{i j} \cdot V(t), \quad N_{R} \cong \frac{|\Delta \vartheta|_{\max }}{\lambda / w}=|\Delta n|_{\max } \frac{2 L_{d}}{\lambda}=\frac{L_{d}}{\lambda} n_{0}^{3} r_{i j}|\Delta V|_{\max },
$$

where $L_{d}$ stands for full length of deflecting device, $w$-is the laser beam width (along direction of deflection), $|\Delta \vartheta|_{\max }$ stands for full variation of deflection angle and electrical field substituted as $E=V / a$. For $L_{d}=25 \mathrm{~cm}$, one can expect for $w \cong a \cong 0.5 \mathrm{~cm}$, that deflection angle is $\Delta \vartheta \cong 10^{-2}$ and $N_{R} \cong 20$ for $\lambda \cong 10 \mu \mathrm{m}$ and, respectively $\Delta \vartheta \cong 10^{-2}$ and $N_{R} \cong 200$ for $\lambda \cong 1 \mu \mathrm{m}$.

We estimated the field amplitude applied to the crystals as $10 \mathrm{kV} / \mathrm{cm}$. This is really a field strength variation along the laser bunch. This variation is traveling together with the laser beam along the sweeping device, Fig. 4.

For acceleration, the laser flash energy passing through the single deflection system is $\sim 10 \mathrm{~mJ}$. We estimated the area cowered by the laser beam as $S \cong w \cdot d$, what is about $0.25 \mathrm{~cm}^{2}$. This yields the energy density $40 \mathrm{~mJ} / \mathrm{cm}^{2}$ only. One can decrease the area at least 10 (up to 100) times. Reduction of orthogonal dimension will reduce the power required for deflection.

For such a sweeping device, a lot of electro-optical crystals can be used, see Table 1 below. For example, a crystal $\mathrm{KDP}\left(\mathrm{KH}_{2} \mathrm{PO}_{4}\right)$ is transparent for a radiation with the wavelength $\lambda \cong 0.2 \div 1 \mu \mathrm{m}$. Some other crystals, such as a $\mathrm{CdTe}, \mathrm{CuCl}, \mathrm{GaAs}, \mathrm{ZnTe}, \mathrm{ZnS}$ are transparent in the region of wavelengths around $\lambda \approx 10 \mu \mathrm{m}$. The last group of materials have rather high refractive indexes $n_{0} \sim(2-4)$ what compensate smaller electro- optical coefficient.

TABLE 1. Electro-optical materials for deflector

\begin{tabular}{|c|c|c|c|}
\hline Wavelength & Materials & $\vartheta$, rad & $N_{R}$ \\
\hline$\lambda \cong 10 \mu m$ & $\mathrm{GaAs}, \mathrm{ZnTe}, \mathrm{ZnS}, \mathrm{CdS}, \mathrm{CdTe}, \mathrm{CuCl}$ & $0.01-0.02$ & 20 \\
\hline$\lambda \cong 5 \mu \mathrm{m}$ & $\mathrm{LiNbO}_{3}, \mathrm{LiTaO}_{3}, \mathrm{CuCl}$ & $0.01-0.02$ & 40 \\
\hline$\lambda \cong 1 \mu \mathrm{m}$ & $\mathrm{KDP}, \mathrm{DKDP}, \mathrm{ADP}, \mathrm{KDA}, \mathrm{LiNbO}_{3}$ & $0.01-0.02$ & 200 \\
\hline
\end{tabular}

Despite the materials transparent for longer wavelengths have lower value of $r_{i j}-$ components, they have higher values of refracting index, so the variation of refractive index becomes about the same. 


\section{PRACTICAL REALISATION}

Let us continue with the description of the deflection mechanism. In Fig. 5, there is represented a side view on scheme with an angular sweeping device. Additional lens 3 has a focal point located in effective sweeping center. After this lens laser bunches have no angular divergence. Lens $3 \mathrm{a}$ has focal point located at the accelerating structure, what is the plane marked 4 . So the sweeping device 2 located between lenses $3 a$ and 3 .

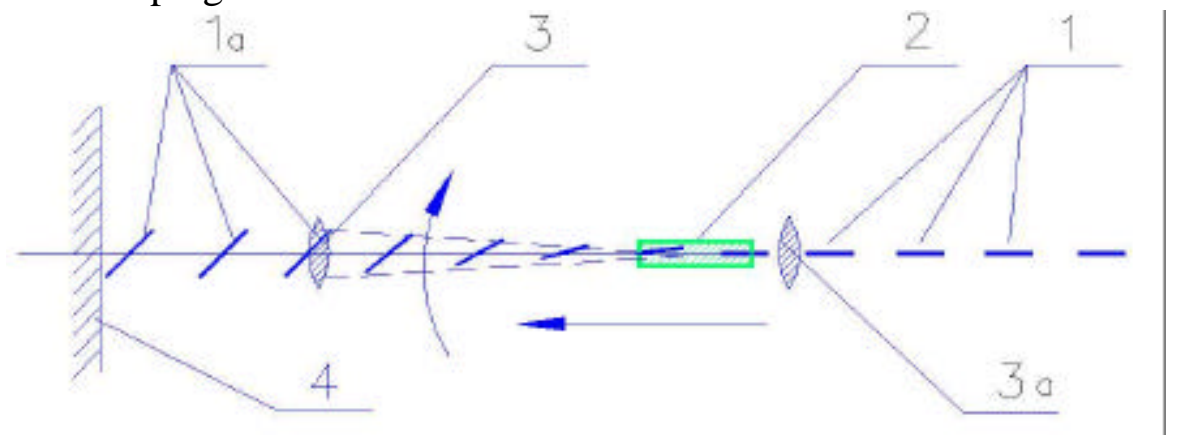

FIGURE 5: Principle of laser bunch sweeping, look from the side direction. 1-shows laser bunch configuration at the entrance, 1a- is a bunch after second lens, 2-is a sweeping device, 3 and $3 a-$ are the focusing lenses.

Direction of sweep defines the laser bunch slope. For practical applications second lens 3 can be combined with cylindrical lens (Fig. 1 and Fig.2). Optimization of sweeping device shows, that its length must be $\cong 2 / 3$ of distance from lens 3 a to the lens 3 , see reference in [3]. In Fig. 6 the further development of this scheme is shown. Here, semitransparent mirrors 8 split controllable fractions of laser radiation and direct it to the accelerating structures. Here the optical amplifier is installed after the sweeping device. By this method there is a possibility to enlarge the volume with active media. It is interesting, that if in this scheme direction of sweep is reversed, the slope of laser bunch at accelerating structure reversed also, so it becomes possible to accelerate particles in opposite direction. The remaining elements described in figure capture. If laser bunch doubler (position 4 in Fig. 2) is omitted, then each next accelerated bunch must be omitted too. In this case effective accelerating gradient come to its value.

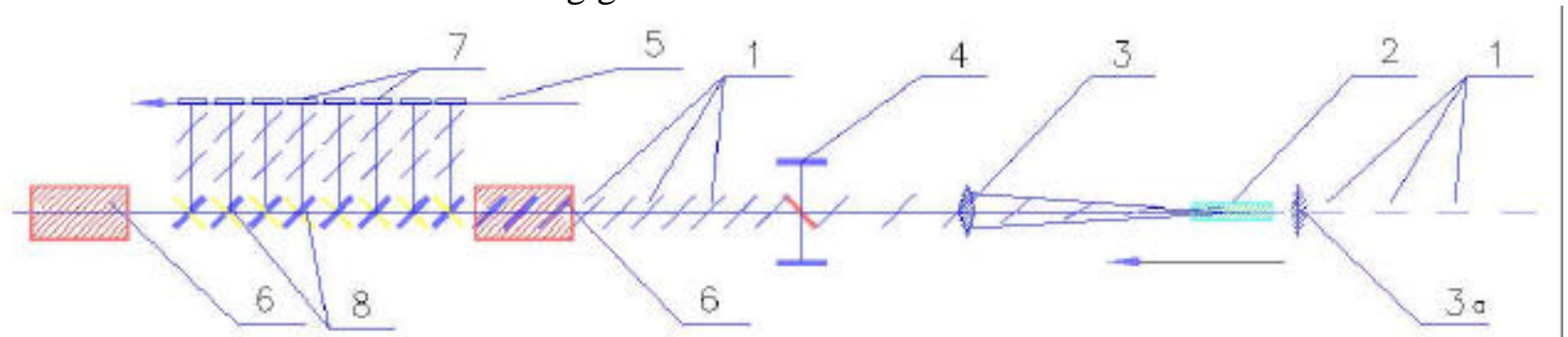

FIGURE 6: Extended scheme with single weeping device. 1-shows laser bunch configuration at different locations, 2-is a sweeping device, 3-focusing lens, 4-laser bunch doubler, 5-accelerated bunch, 6-optical amplifier, 7-accelerating structures, 8-power splitters (semi-transparent mirrors).

Let us make one comment about one peculiarity on how sloped laser bunch reflects from the tilted mirror. This process is shown in Fig. 7, where swept laser bunch shown in four different moments of time, marked by 1-4. Laser bunch is coming from the right side in Fig. 7. At the left, the bunch is swept so that its head located at the right side (to the direction of motion). At the right, it is swept in opposite way. 


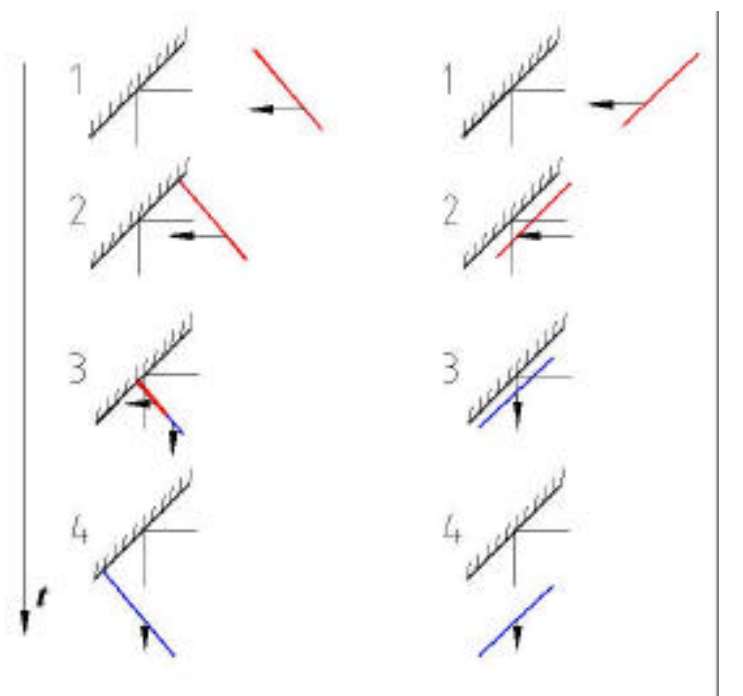

FIGURE 7: Reflection of sloped laser bunch from a mirror. Bunch tilted oppositely to the mirror slope, left, and tilts in same direction as the mirror slope, right. Numbers indicate moments of time. Arrows show direction of propagation. Incoming bunch colored in red, reflected one is colored in blue.

One can see that reflection from single mirror is going with reverse of the slope direction with respect to direction of propagation. One peculiarity here is that if the bunch and mirror surface does not have the same slope (left side in Fig. 7), then electromagnetic radiation from incoming and reflected beams is overlapped. This is shown in position 3, left side.

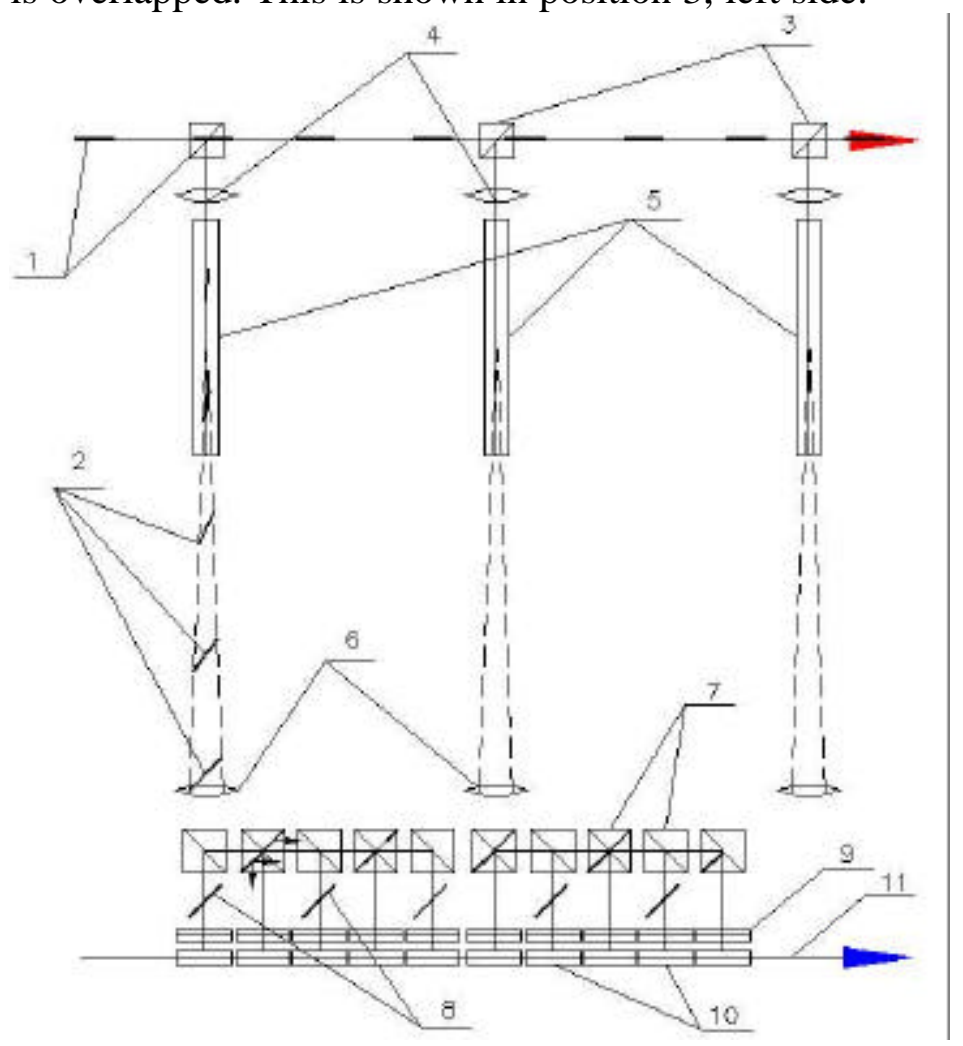

FIGURE 8: Sweeping device serves for few accelerating structures. Comments are in the text. 
In Fig. 8 the laser bunch train, 1 coming from the left and passing sequentially power splitters 3. By 2 marked locations and configuration of the swept laser bunches. Lenses 4 installed a prior to the sweeping devices 5 having focal plane at location of lens 6 . By 7 marked power splitters and mirrors allowing feed few structures from single sweeping device. Even number of reflections (basically two), bring the slope to the proper tilt shown by 8 . This system also equipped by cylindrical lenses 9 which have transverse focus on the openings of accelerating structures. Structures marked by 10. Accelerated bunches are running to the right 11 inside structures.

So mostly progressive looks the way of feeding few structures from one sweeping device. The number of devices is limited to the depth of focus provided by lens 4 . This means that Rayleigh length for the focusing length required must be longer, than the difference in pathways for these structures. We expect that this can be done for 5-10 structures.

\section{ENGINEERING}

First off, let us consider the schemes described above, transforming them into a more realistic hardware view. We begin with the scheme represented in Fig. 6 first, although this sweeping device can be used in any modification.

\section{Waveguide sweeping device}

So the slope of High Voltage must be $\sim 10 \mathrm{KV}$ at the distance $\sim c \tau \cong 3 \mathrm{~cm}$. Naturally UHF electromagnetic waves satisfy the requirements. These types of waves having period $\sim 2-3 c \tau$ what comes to $\sim 5-10 \mathrm{~cm}$. In this case the waveguide is mostly the natural element to use. Stripline is also possible here and it becomes more desirable as it allows to make the sweeping device more compact, closer to integrated cirquit. Let us estimate the power requirements and possible design of waveguide-based sweeping device. Example of such a device is represented in Fig.9.

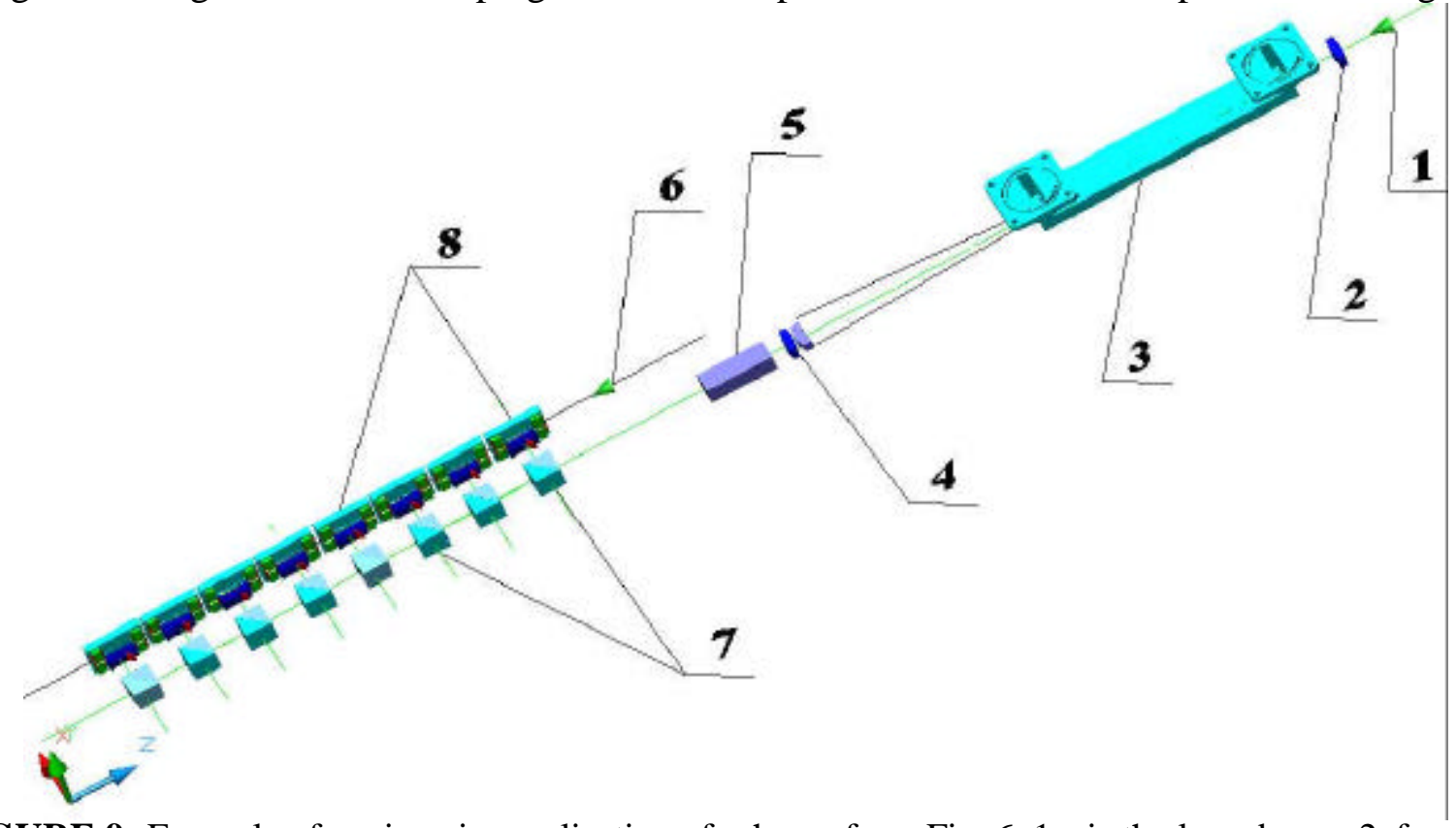

FIGURE 9: Example of engineering realization of scheme from Fig. 6. 1 -is the laser beam, 2-focusing lens, 3-waveguide sweeping device, 4-lens, 5-optical amplifier, 6-particle beam under acceleration, 7-laser power splitting devices, 8-accelerating structures with beam focusing elements. 
The broad band traveling wave deflector uses crystals located in the middle of a waveguide. Group velocity of electrical wave-pulse must be adjusted to the velocity of the laser radiation in the prisms. Widening of the waveguide helps to reach this goal. Additional dielectric, 6 in Fig 10 , serves for this purpose.

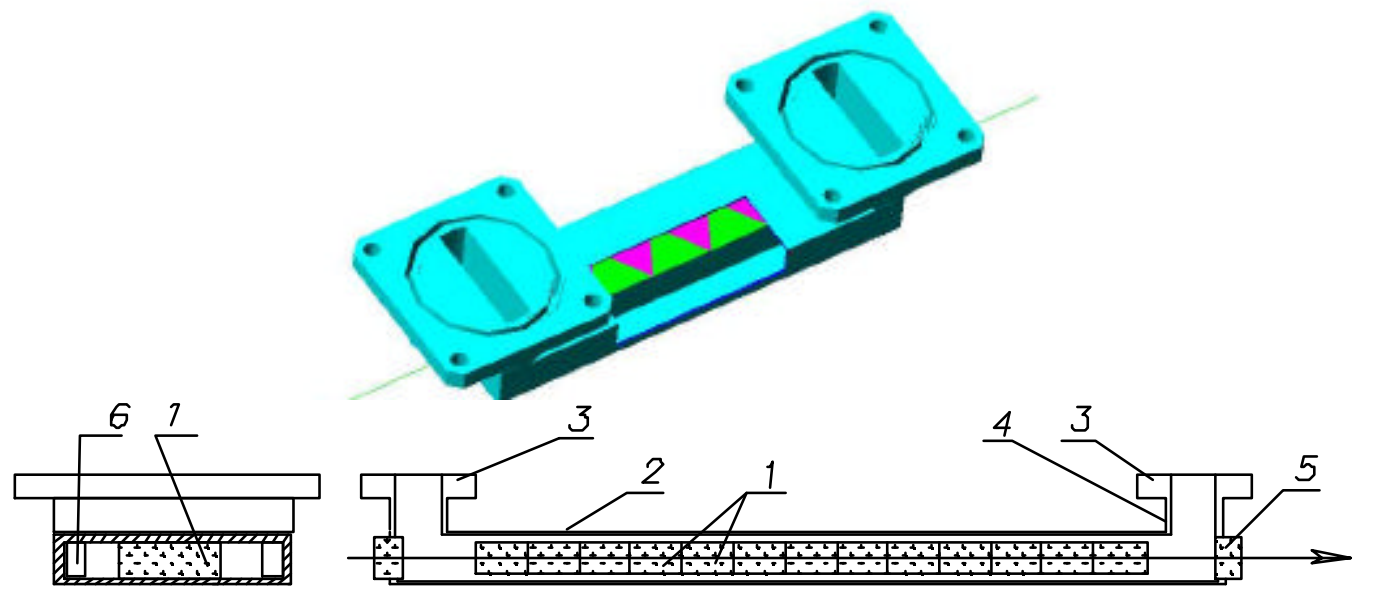

FIGURE 10: Multi-prism traveling wave sweeping device in a waveguide. 1-is electro-optical crystals, positioned in a waveguide 2, having bends 4 with flanges 3. 5-is an optical window. 6 -is a matching dielectric.

For waveguide deflector a klystron generator is mostly suitable power source. In a waveguide having cross-section $a \times b$ for the fundamental mode $H_{10}$, the electric field excited has a form $E=E_{0} e^{i \beta z}$, where propagation constant defined as

$$
\beta=\sqrt{\omega^{2} \varepsilon_{e f f}-\left(2 \pi / \lambda_{c r}\right)^{2}},
$$

and $\varepsilon_{\text {eff }}$ stands for effective dielectric permittivity of the waveguide, $\omega$ is frequency and critical wavelength $\lambda_{c r} \cong 1 /\left(2 a \sqrt{\varepsilon_{e f f}}\right)$. For this type of wave the power transmitted and electrical field strength can be expressed as a relation

$$
P \cong \frac{E_{0}^{2} a b}{4 Z_{0}} \sqrt{1-\left(\lambda / \lambda_{c r}\right)^{2}},
$$

where $Z_{0} \cong 120 \pi \sqrt{\varepsilon_{\text {eff }}}$. Substitute here $a \cong 5 \mathrm{~cm}, b \cong 1 \mathrm{~cm}, \lambda \cong 5 \mathrm{~cm}, E_{0} \cong 20 \mathrm{kV} / \mathrm{cm}$ one can obtain the power, running through, as $P \cong 1.2 \cdot 10^{6}$ or $1.2 \mathrm{MW}$, what is small enough.

For further power reduction required from the RF source, the resonant O-ring system can be used here. For this purposes "in" and "out" flanges connected, and directional coupler excites the traveling wave in this ring, see Fig. 11. If we suggest the quality factor for O-ring about modest value $\sim 100$, then power required for excitation goes to be $\sim 1 \%$ of $1 \mathrm{MW}$. So RF generator can feed many devices in this way.

Time structure of the accelerated beam now can be seen as the train of bunches spaced with period $\sim$ wavelength of sweeping $\mathrm{RF}$ and these trains running with repetition rate $\sim \mathrm{few} \mathrm{kHz}$, i.e. regime pretty typical for Radar operation. For the duty of RF $\sim 1 \mu \mathrm{s}$, the number of bunches in the train can be estimated as $n \cong 1 \mu s / 200 p s=5000$. As these bunches running with repetition rate $\sim 1 \mathrm{kHz}$, this brings the number of bunches per second to $n_{b} \cong 5 \cdot 10^{6}$. 


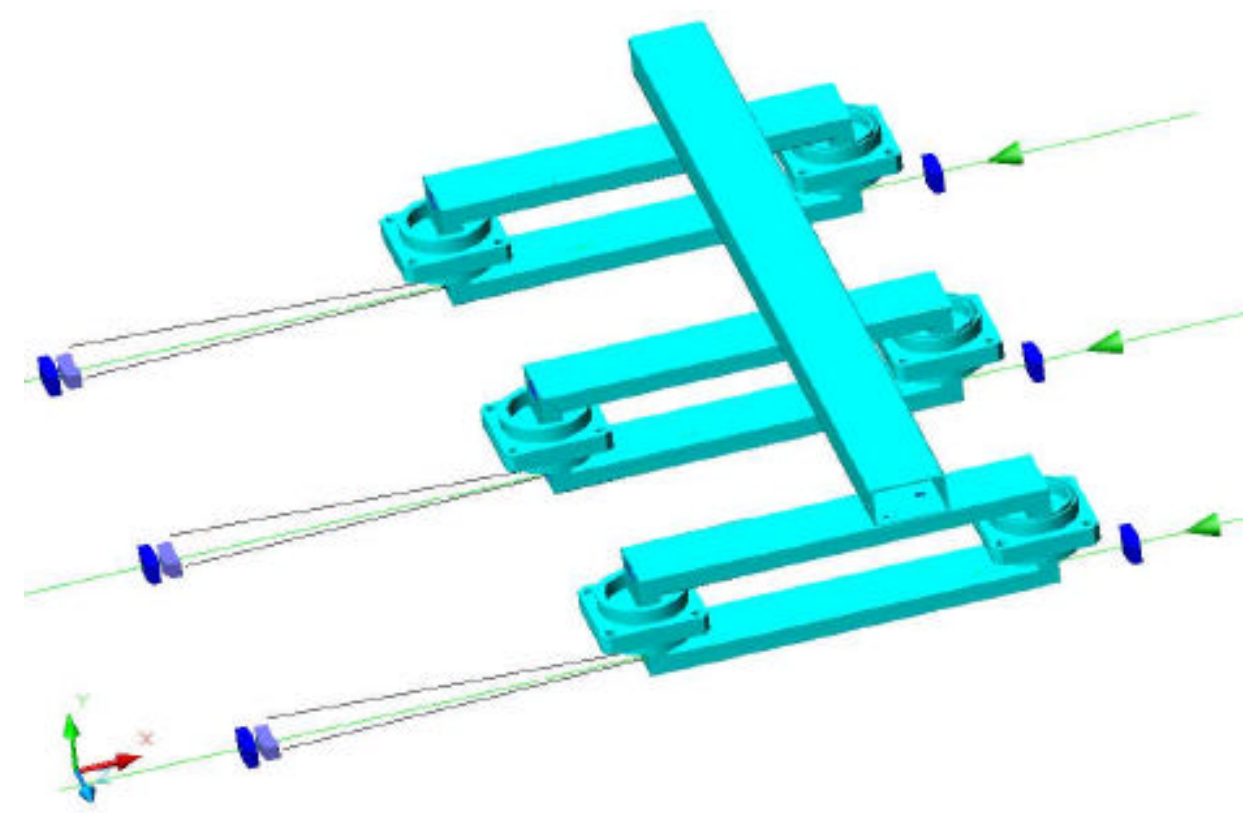

FIGURE 11: Feeding of sweeping devices in parallel. Upper waveguide running through full length of accelerator excites traveling wave in each O-ring resonators.

Once again, this scheme has a potential to be transformed into strip-line system. O-ring systems are well known in strip-line realization of transferring RF systems.

\section{Sweeping device with pulsed voltage}

Example of technical realization of this traveling wave deflector as a strip-line is represented in Fig. 12. Here, basically, strip-line electrodes 1 have triangle crystals 3 with opposite optical axes orientation in between. HV impulse applied to one end of strip-line trough connectors 4 , propagates to the other end and further connected to the matching resistor 2 . Slots in electrodes 1 made for proper current distribution across the line taking into account that wave front $\sim c \tau$ becomes comparable with the transverse size of the line.

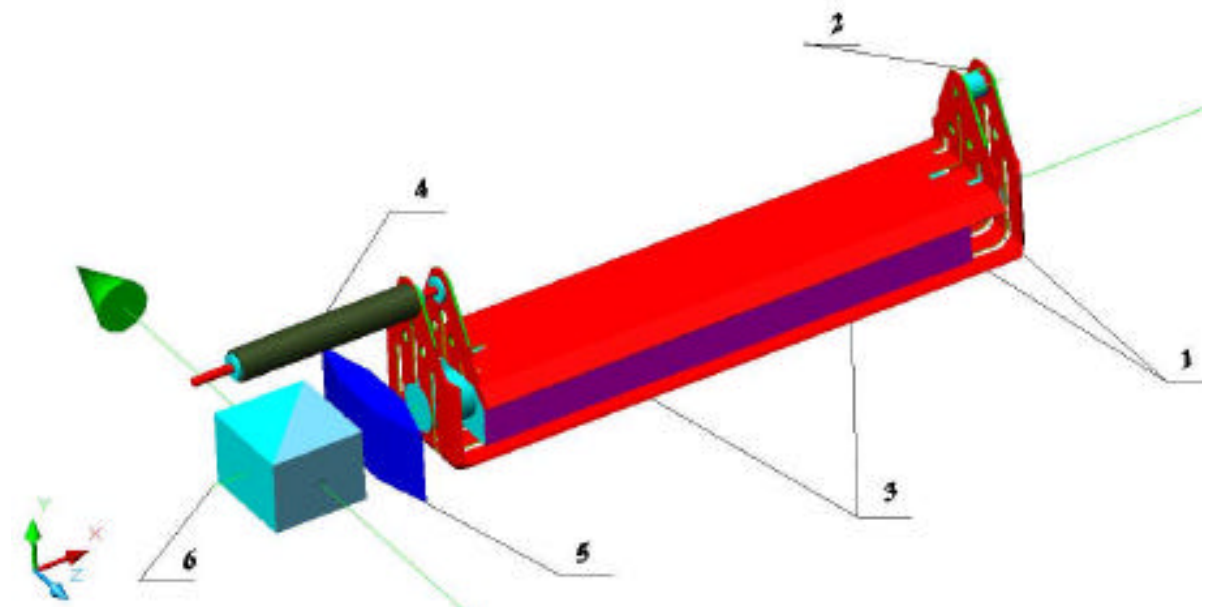

FIGURE 12: Example of technical realization of multiprism sweeping device with traveling wave and pulsed feeding. Electro-optical crystals 3 are positioned between strip-lines 1, attached at the end to the matching resistors 2. Excitation is going with the help of cable 4.5 and 6 are lens and splitting device respectively. 
In Fig. 12 is shown the lens, 5 giving longitudinal focus to the swept laser beam and the power splitter 6 which splits a fraction of laser radiation propagating along the accelerator.

This engineering realization is very suitable for testing this principle. Powering of this device can be arranged with the power generator using for its operation Inversely Recovered Diodes technique [4], [5]. In these types of techniques, energy stored in an inductor while the current running through it. While fast-interrupted, this current flow raises voltage on the load. For interruption of the current the Drift-Step Recovery Diodes (DSRDs) used successfully. In more detail, the transition filled by the carriers during direct charge flow first. Then the second pulse with opposite polarity runs through the transition during the time determined by evacuation of carriers (electron-hole plasma) accumulated in transition region.

Typical PS of this class operates with repetition rate up to $1 \mathrm{MHz}$ providing rise time down to $50 \mathrm{ps}$ and voltage up to $30 \mathrm{kV}$. Size of this device typically $350 \times 150 \times 300 \mathrm{~mm}^{3}$.

\section{Optical triggering}

A more advanced device is shown in Fig. 13. Here a Laser switched diode serves as the key element in triggering high voltage. First possibility might utilize the filling of diode transition by carriers with the help of laser pulse illuminating the diode. The second possibility lies in fast changing dielectric permeability of switching element by the laser radiation.

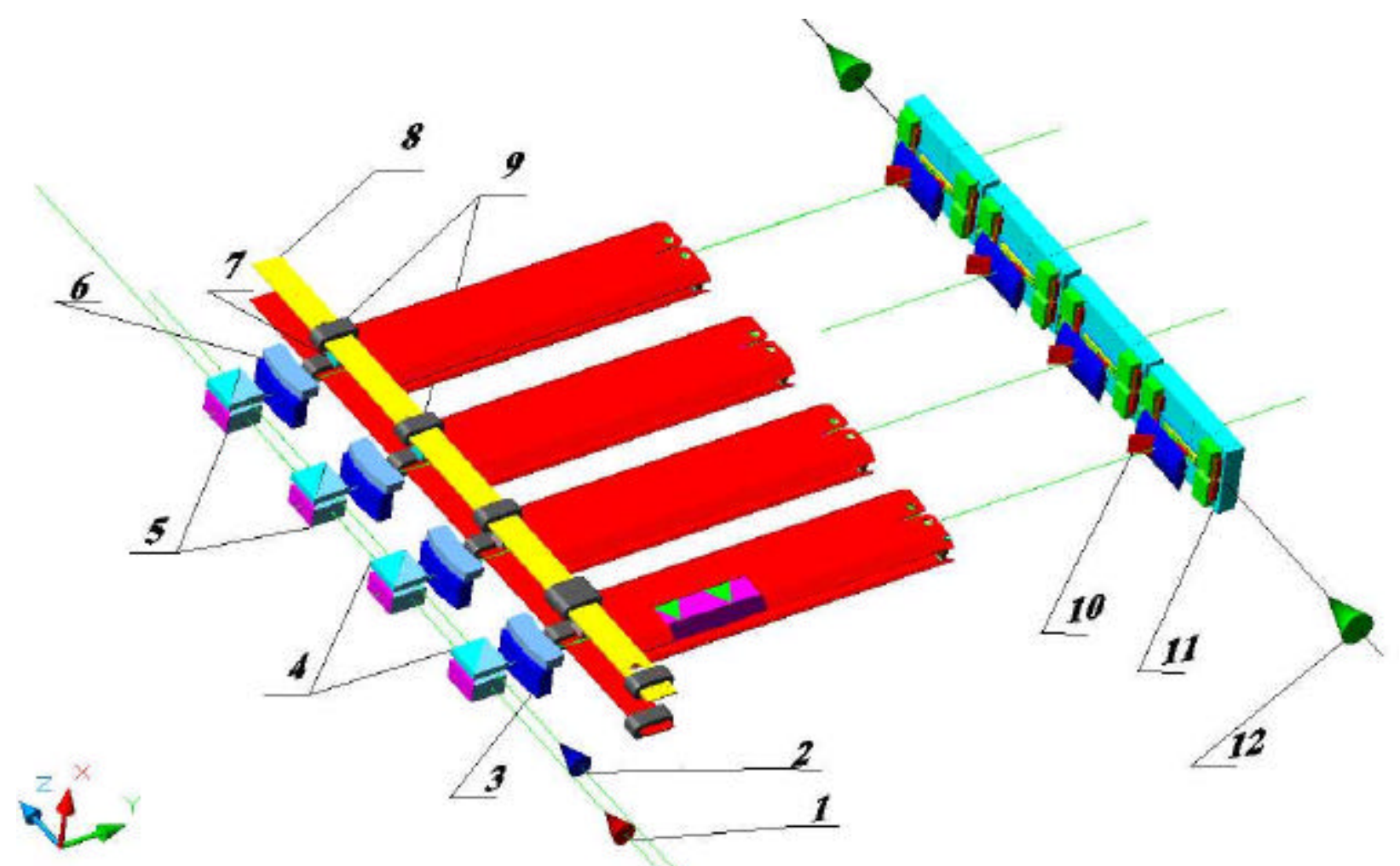

FIGURE 13: In this scheme triggering is going by additional laser bunch 2 .

Here in Fig. 13, two laser pulses, marked by 1 - for main accelerating pulse and by 2 - for the triggering one are propagating in the same direction. Lenses 3 focuses main laser pulse on accelerating structure plane (marked 11) and short focusing lenses 6 focus laser pulse onto triggering element 7. Splitters 4 and 5 serve for acquiring fraction of laser energy both from 
triggering and main (accelerating) laser pulses. Energy store in line 8 divided by inductors 9 into short pieces. Each of these pieces is associated with its own sweeping device. The strip-line, marked red (see also Fig.12) feed by this piece of line. While illuminated by triggering laser pulse the triggering element 7 transferees the energy stored in short piece of line to the sweeping strips. By 10, 11 and 12 the laser bunch configuration, accelerating structure module and accelerating bunch trajectory marked respectively. Synchronization required is now severe as the only phasing important for acceleration is the phase of main laser pulse, which is naturally the same over all accelerator. Despite of more advanced possibilities for integration of these elements into unite module, this scheme requires some development and mentioned here as a direction for future activities.

\section{CONCLUSIONS}

In spite of these unique properties, the device for the laser sweep requires, for its realization, well developed elements. A waveguide deflection device is looking absolutely guaranteed. Other possibility associated with usage of DSRD technique. Both RF generators for waveguide sweeping device and pulsed generators based on DSRD's for feeding the sweeping device widely available on the market.

A high repetition rate is achievable with waveguide sweeping device allowing $\sim 10^{6}-10^{7}$ bunches per second opens broad variety for optimization of luminosity with colliding beams.

\section{REFERENCES}

[1] A.A.Mikhailichenko, "The Method of Acceleration of Charged Particles", Author's certificate USSR N1609423, Priority May 1989, Bulletin of Inventions (in Russian), N6, p.220, 1994.

[2] A.A. Mikhailichenko, "Physical Foundations for Acceleration by Travelling Laser Focus", Presented at AAC Workshop, Stony Brook, NY June 21-26, CBN 04-6 ${ }^{3}$.

[3] A.A.Mikhailichenko, "Particle acceleration in Microstructures, Excited by Laser Radiation”, CLNS 00/1662, Cornell 20004; Also Snowmass 2001. Full list of referred can be found there.

[4] I.V.Grekhov, V.M. Efanov, A.F. Kardo-Sysoev, S.V. Shenderei, Pis'ma Zh.Tech.Fiz., 1983, vol.9, no 7, p.435.

[5] I.V.Grekhov, V.M. Efanov, A.F. Kardo-Sysoev, S.V. Shenderei, Solid-State Electron., 1985, vol.28, no 6, p.597.

\footnotetext{
${ }^{3}$ Electronic version available at http://www.Ins.cornell.edu/public/CBN/2004/CBN04-6/phys_found.pdf

${ }^{4}$ Electronic version available at http://www.Ins.cornell.edu/public/CLNS/2000/
} 